\title{
Proyecto docente innovador desarrollado en Grado en Ingeniería en Diseño Industrial y Desarrollo de Producto para la consecución de un Prototipo Funcional mediante trabajo en equipo
}

Aroca Martinez, Antonio; Calles Díaz, Héctor; Chisbert Victory, Daniel; Cremades Navarro, Miguel Ángel; Diago De Rozas, Rodrigo; Esplugues Calabuig, David; Ferez Navarro, Mario; González Simón, Francisco Javier; Morera Gilabert, Óscar; Munguía Lozano, María Fernanda; Ortiz Castillo, Álvaro; Ortuño Ayuso, Pablo; Pérez Montoya, Sergio; Popescu, Gabriel; Requena Felis, Ángel; Revuelta Arnao, Paula; Such Savall, Cristian; Talens Payá, Adrián; Vega Rubio, Tamara; Verdú Calvo, Carlos; López Martínez, Juan; Rayón Encinas, Emilio*.

Departamento de Ingeniería Mecánica y Materiales. Escuela Politécnica Superior de Alcoy. Universitat Politècnica de Valencia. Plaza Carbonell s/n. Alcoy, Alicante.

\begin{abstract}
Resumen
Se propone un método docente innovador para fomentar el trabajo en equipo a la vez que se adquiere habilidades en nuevas tecnologías de interés para el ingeniero de diseño industrial y del producto. Inicialmente a los estudiantes se les propone realizar una investigación siguiendo una metodología de aprendizaje inverso, invitándoles a analizar experimentalmente cómo funcionan muchos de los aparatos tecnológicos que los rodean. Se trata de redescubrir la curiosidad innata de aprendizaje desmontando un producto para ver de qué está hecho y qué mecanismos lo hacen funcionar. Además, durante estas jornadas teórico-prácticas se les muestra materiales, instrumentos y metodologías para realizar prototipos funcionales, como son materiales luminiscentes, escáneres $3 D$, arcillas de modelado y tecnologías novedosas de código abierto dedicadas al prototipado.
\end{abstract}

Las posteriores evaluaciones y la experiencia adquirida con esta metodología demuestra que la metodología implantada consigue favorecer el trabajo en equipo a la vez que se recupera el entusiasmo por un aprendizaje nutrido desde la curiosidad. La mayoría de los estudiantes coincidieron en lo valioso que les resultó el conocimiento adquirido, incluso han podido aprovechar estas experiencias para desarrollar un modelo funcional para sus respectivos $P F G$.

Palabras clave: código abierto, Arduino, Diseño, prototipado, prototipo funcional

\footnotetext{
Abstract

The aim of this project was to make a technological and functional prototype. This innovative methodology was developed in the Engineering and Industrial Design and Product Development, inside the mention of
} 
Proyecto docente innovador desarrollado en Grado en Ingeniería en Diseño Industrial y Desarrollo de Producto para la consecución de un prototipo funcional mediante trabajo en equipo

\begin{abstract}
Production and Innovation. It counts whit an innovative methodologies to show teaching methods so the students interact with a real external university environment to present projects, ideas and proposals made by the students.
\end{abstract}

\author{
Keywords: Methodology, Innovation, Technology, Education, Reverse \\ Engineering, Open Source.
}

\title{
1.- Introducción
}

La metodología que se describe ha sido desarrollada en la asignatura de Tecnología del Producto que viene cursándose en el último curso del grado en Ingeniería en Diseño Industrial y Desarrollo de Producto de la Universitat Politècnica de València en el Campus d'Alcoi (EPSA). El objetivo de este grado es dotar al estudiante y futuro ingeniero de los recursos científico y técnicos necesarios para dirigir y gestionar todo el proceso de desarrollo de producto, desde la generación de ideas, diseño, prototipado y producción.

Según se ha podido constatar en el mercado laboral existe un creciente interés en disponer de diseñadores con capacidades técnicas capaces de llevar a cabo prototipos físicos de los objetos que están en fase de desarrollo. La virtud de disponer de un modelo real de lo que se está diseñando sobre una mesa de dibujo o por ordenador, es poder hacerse una idea tangible de lo que se va a producir, teniendo una percepción más real que si el modelo fuera representado en la pantalla de un ordenador. Esto permite tomar decisiones que puedan afectar al diseño final antes de emprender el trabajo de producción y por lo tanto ahorra costes y tiempo. Se ha comprobado que estos modelos reales permiten detectar fallos o detalles que de otro modo hubiera sido más difícil de contemplar, permitiendo la toma de decisiones en un momento de desarrollo oportuno. Como valor añadido, el disponer de un modelo real permite realizar laborales comerciales que sirven como sondeo de mercado y venta.

Cabe aclarar que el prototipo de un objeto viene realizándose de manera casi natural por muchos departamentos de empresas de este sector, sin embargo en la actualidad han surgido nuevas tecnologías que actualmente están revolucionando la manera de desarrollar prototipos. Las tecnologías más destacadas en este sector son el escaneado e impresión 3D y los sistemas electrónicos de código abierto. Las primeras permiten replicar un objeto real siguiendo un modelo generado por ordenador. Las segundas, también llamadas tecnologías 'open source' se fundamentan en proporcionar herramientas electrónicas a un precio muy asequible en comunidades abiertas donde toda la información y experiencia es compartida con ayuda de plataformas en internet. De este modo, todo el desarrollo que se genera en el mundo con esta tecnología es accesible a golpe de 'click' y puede ser aprovechado, modificado y mejorado con mucha facilidad. Esta cantidad ingente de conocimiento crece y se comparte a nivel mundial de forma continua.

Por otro lado, también es cada vez más requerida la capacidad de trabajar en equipos multidisciplinares, donde cada profesional tiene que compartir de manera fluida mucha información con otros departamentos implicados en el desarrollo del producto. Amén de la inteligencia emocional necesaria para ser proactivo a la vez que operativo y funcional en un equipo de trabajo multidisciplinar, es importante que el diseñador tenga los conocimientos básicos necesarios para entender y hacerse entender con el resto de áreas implicadas en la creación del producto y es por eso que al ingeniero de diseño se le forma durante su grado

(c) EY-NC-ND 2016, Universitat Politècnica de València 
Aroca Martinez, Antonio; Calles Díaz, Héctor; Chisbert Victory, Daniel; Cremades Navarro, Miguel Ángel; Diago De Rozas, Rodrigo; Esplugues Calabuig, David; Ferez Navarro, Mario; González

Simón, Francisco Javier; Morera Gilabert, Óscar; Munguía Lozano, María Fernanda; Ortiz Castillo, Álvaro; Ortuño Ayuso, Pablo; Pérez Montoya, Sergio; Popescu, Gabriel; Requena Felis, Ángel; Revuelta Arnao, Paula; Such Savall, Cristian; Talens Payá, Adrián; Vega Rubio, Tamara; Verdú Calvo, Carlos; López Martínez, Juan; Rayón Encinas, Emilio.

en áreas tan diversas como la de ciencia de materiales, software de modelado y diseño, física, propiedades mecánicas de sólidos, química y electrónica.

En la asignatura Tecnología del Producto, según el POD establecido para la misma, el estudiante debe de adquirir precisamente habilidades y conocimientos para poder desarrollar trabajos futuros en prototipados funcionales y en resolución de proyectos de diseño de un producto mediante toma de decisiones en equipo.

Con todo esto en mente, proponemos aquí un método que hemos experimentado durante el curso 2015-2016 en la asignatura de Tecnología del Producto que pensamos ha sido muy enriquecedor tanto por su valor didáctico como por lo innovador del propio método.

\section{2.- Objetivos}

Son varios los objetivos que se esperan conseguir mediante la metodología descrita. Por un lado se pretende que los alumnos redescubran la capacidad innata de aprendizaje mediante el descubrimiento y la experimentación utilizando un método de aprendizaje inverso en el que deberán aprender con el ejercicio de preguntarse ¿Cómo funciona esto? Se pretende además que conozcan nuevos materiales de modelado y tecnologías novedosas que les sirva para realizar un prototipo de un producto. Siempre se tendrá en cuenta que los materiales utilizados en el aula sean muy accesibles para el estudiante si éste desea adquirirlo en el mercado. Para finalizar, como material evaluable, los alumnos realizarán un proyecto en grupo con el fin de alcanzar un prototipo funcional: un objeto que emita sonidos y realice movimientos en función de los estímulos que se le apliquen.

\section{3.- Desarrollo de la innovación}

La metodología que se describe fue aplicada a un grupo de 24 estudiantes con una duración total entre sesiones teóricas y prácticas de 12 horas lectivas. A continuación se describe la metodología descrita describiendo las tareas y materiales utilizados según el orden cronológico desarrollado en el aula, con el fin de que cualquier docente pueda reproducirlo si lo ve conveniente.

En la primera sesión se muestra al estudiante las diferentes tecnologías que han existido a lo largo de la historia así como las actuales y en proceso de desarrollo para generar luz. No se pretende narrar la historia de la lámpara, sino las formas de las que dispone un ingeniero para producir luz en el producto. Durante la sesión teórica apoyada con imágenes proyectadas se intercalan ejemplos reales en la clase. El docente tiene preparado algunas lámparas antiguas así como novedosos sistemas y materiales que emiten luz.

Durante la clase se conectan estos dispositivos, comprobando cómo antiguas lámparas de cátodo caliente o de descarga luminiscente, ahora vuelven a ponerse de moda para desarrollar objetos con diseño 'vintage'. En la figura 1 se muestra una fotografía tomada en clase donde se aprecia una lámpara de gas neón antiguamente utilizada para tratamientos

(c)) EY-NC-ND 2016, Universitat Politècnica de València

Congreso In-Red (2016) 
médicos. A los estudiantes les llama aún más la atención materiales novedosos como son los tubos de cátodo frio o los materiales flexibles luminiscentes. La figura 2 muestra las fotografías de los hilos flexibles y electroluminiscentes con los que los alumnos pudieron experimentar. A medida que iban trabajando con estos materiales era evidente, tanto el entusiasmo que mostraban al descubrir estas tecnologías, como la cantidad de ideas que ellos mismos iban proponiendo para aplicar estos sistemas en sus proyectos, diseños, etc.

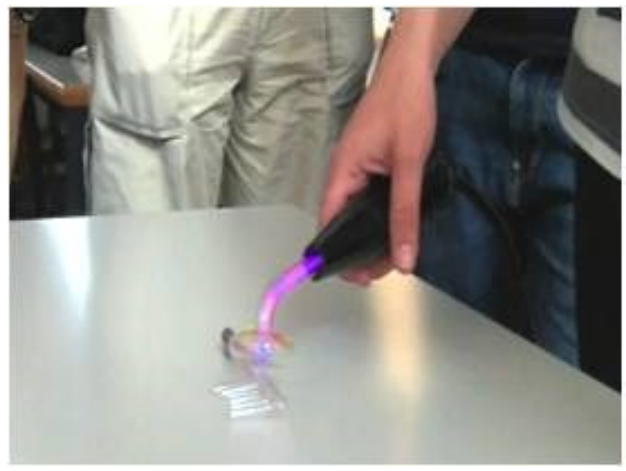

Figura 1. Instrumento medical violet ray, 1915 utilizando durante la clase.

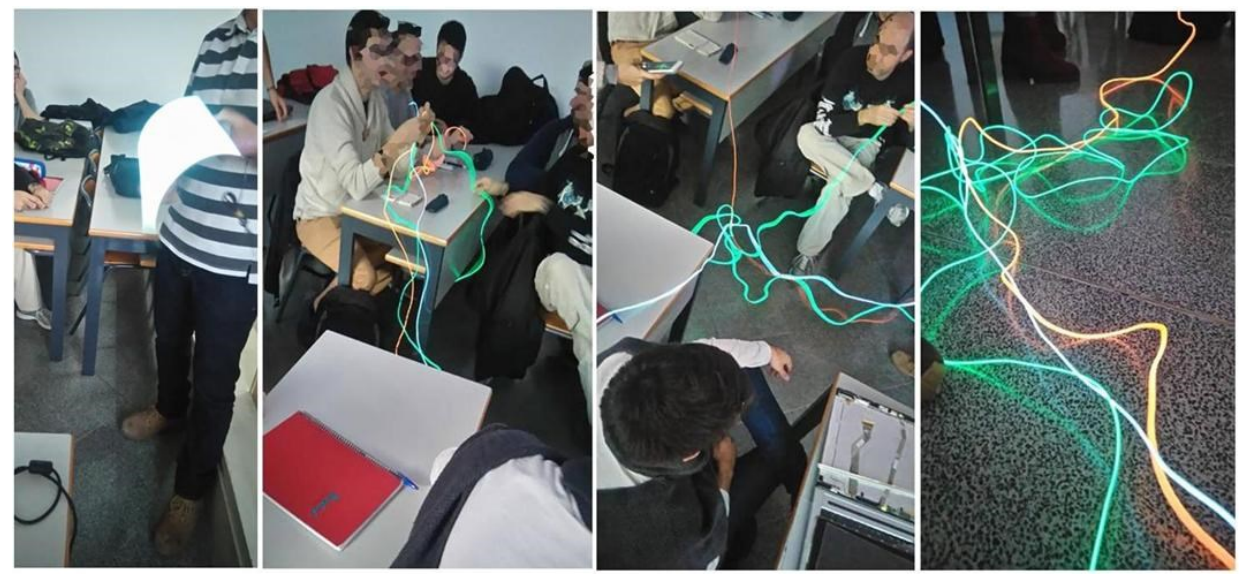

Figura 2. Hojas e hilos flexibles electroluminiscentes de fósforo utilizadas durante la clase

Con el objetivo de que el estudiante reflexione sobre cómo se genera luz en los dispositivos que utilizan diariamente se propone que durante la clase dediquen un tiempo a desmontar la pantalla de un ordenador portátil. Con esta tarea se consigue dos cosas; por un lado se les motiva a hacerse preguntas de cómo funciona el mundo que les rodea con el fin de obtener información que sin duda les será útil en su vida tanto personal como profesional. Como guinda del pastel, encuentran que la pantalla es iluminada mediante un tubo del cátodo frío ubicado en la base de la pantalla, como muestra la figura 3. Se describe el funcionamiento de esta tecnología y dedicamos unos minutos para proponer utilidades y aplicaciones de su interés. 
Aroca Martinez, Antonio; Calles Díaz, Héctor; Chisbert Victory, Daniel; Cremades Navarro, Miguel Ángel; Diago De Rozas, Rodrigo; Esplugues Calabuig, David; Ferez Navarro, Mario; González Simón, Francisco Javier; Morera Gilabert, Óscar; Munguía Lozano, María Fernanda; Ortiz Castillo, Álvaro; Ortuño Ayuso, Pablo; Pérez Montoya, Sergio; Popescu, Gabriel; Requena Felis, Ángel; Revuelta Arnao, Paula; Such Savall, Cristian; Talens Payá, Adrián; Vega Rubio, Tamara; Verdu Calvo, Carlos; López Martínez, Juan; Rayón Encinas, Emilio.

Una vez se ha experimentado con todas las fuentes de luz que el docente ha descrito y llevado al aula, se presentan materiales que pueden combinarse con éstos para generar efectos luminosos espectaculares. Actualmente, uno de los productos que está dedicando más esfuerzo en investigación en aplicaciones ópticas, tanto de objetos tecnológicos como en arquitectura, es el polimetil de metracrilato de Plexiglás ${ }^{\circledR}$ ahora suministrado y representado por ThyssenKrupp Ibérica, empresa situada en Valencia.

El docente contacta con esta empresa que desinteresadamente suministra material de demostración. Además nos cuentan que actualmente Plexiglás dedica mucha inversión en investigación para generar metacrilato especial para diferentes fuentes de luz, como por ejemplo la luz LED. Este material Plexiglás se reparte entre los estudiantes que aún conservan las fuentes de luz conectadas. De manera innata los estudiantes comienzan a realizar combinaciones de efectos luminosos muy aparentes y llamativos, tal y como se aprecia en las fotografías de la figura 4.
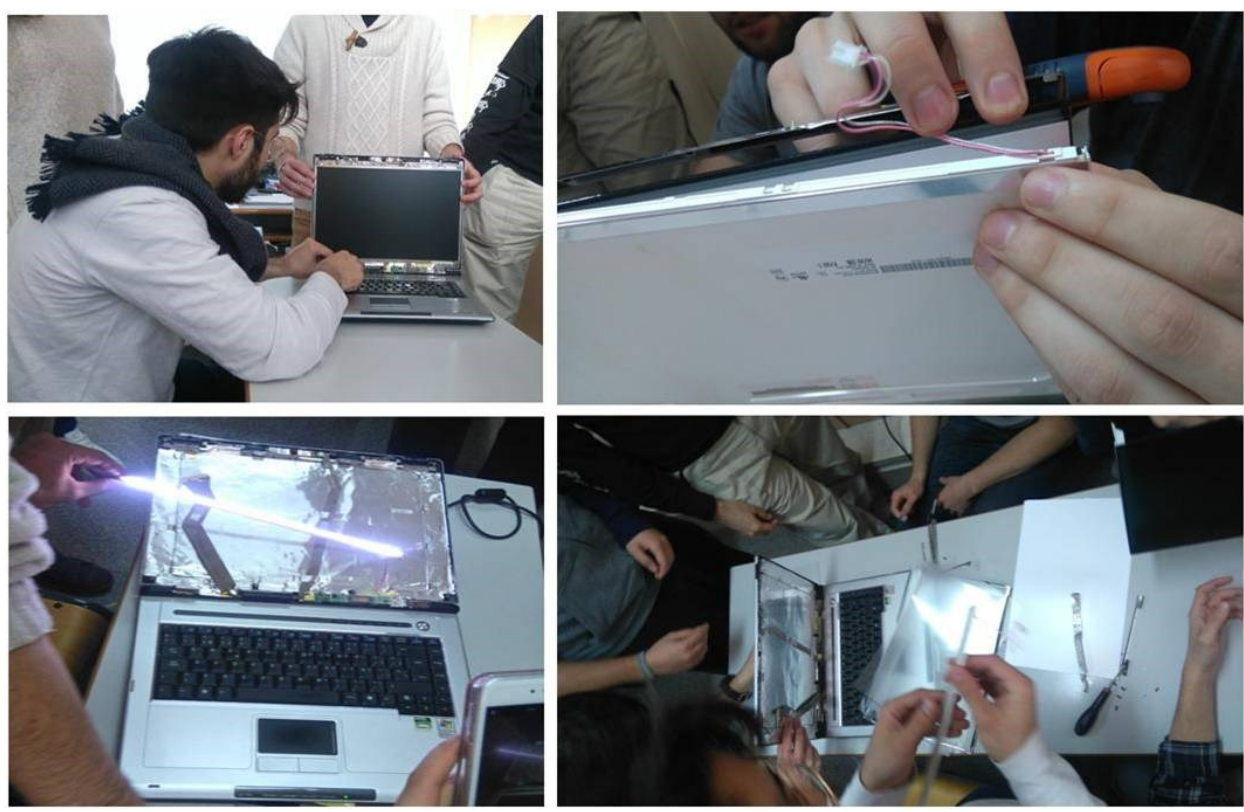

Figura 3. Desmontando la pantalla de un ordenador portátil para llegar al tubo de cátodo frío de un portátil.

(cc) EY-NC-ND 2016, Universitat Politècnica de València

Congreso In-Red (2016) 


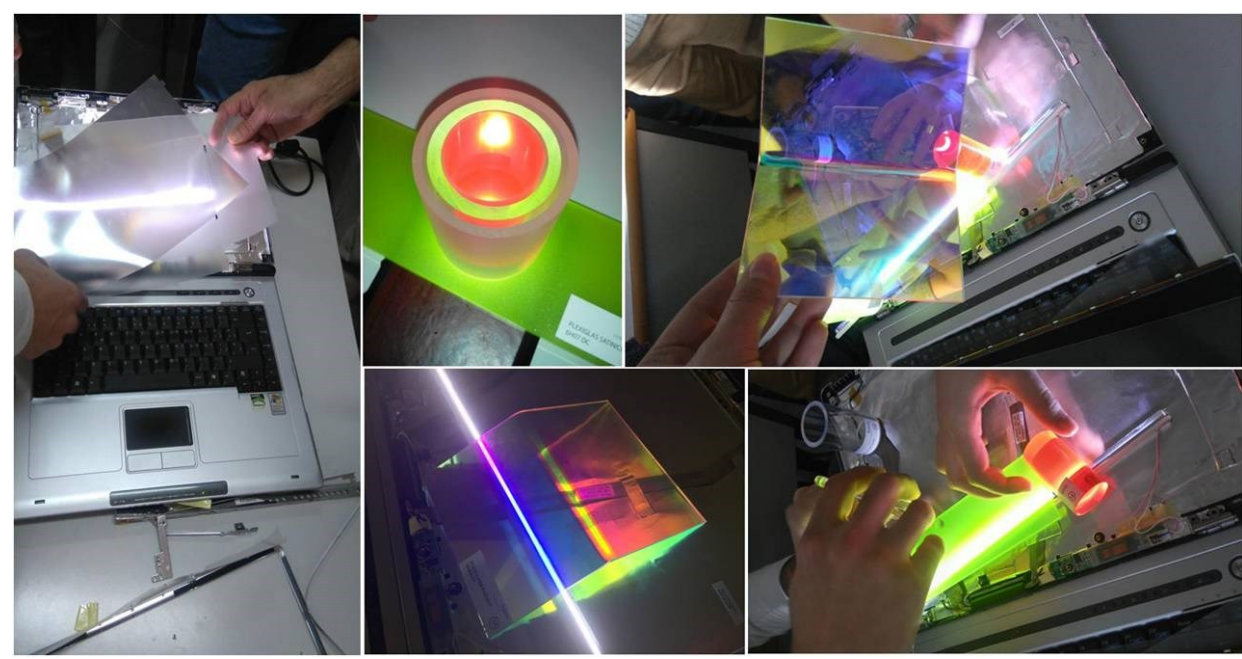

Figura 4. Tubo de cátodo frío extraído del portátil y en funcionamiento. Se experimenta con el PMMA de varios colores para conseguir diferentes efectos lumínicos

En una nueva sesión práctica con una introducción teórica, se comentan materiales profesionales utilizados para desarrollar modelos tridimensionales mediante el modelado con las manos. Realizar un modelo con las manos, como hacen los alfareros con sus objetos permite 'sentir' los volúmenes y formas del objeto, resultando muy fácil modelar de manera libre o mediante patrón de referencia cualquier objeto por complejo que este sea. El resultado es un modelo 3D que permite al modelador ver de forma real el resultado de sombras y de volumen. Esta forma de trabajar la utilizan mucho los diseñadores de automóviles porque resulta más real la sensación de profundidad que el modelo generado por una renderización por ordenador. En esta sesión, se reparte arcilla profesional de modelar a todos los alumnos y se les deja experimentar con sus manos y herramientas para generar un prototipo. Después de realizar algunos modelos de manera individual se pusieron en común ideas para realizar una tabla de inputs y outputs sobre el dispositivo que se deseaba prototipar, que en este caso fue un juguete. La figura 5 y figura 6 muestra algunas fotografías adquiridas durante el modelado de prototipos utilizando la arcilla de modelado profesional. 
Aroca Martinez, Antonio; Calles Díaz, Héctor; Chisbert Victory, Daniel; Cremades Navarro, Miguel Ángel; Diago De Rozas, Rodrigo; Esplugues Calabuig, David; Ferez Navarro, Mario; González

Simón, Francisco Javier; Morera Gilabert, Óscar; Munguía Lozano, María Fernanda; Ortiz Castillo, Álvaro; Ortuño Ayuso, Pablo; Pérez Montoya, Sergio; Popescu, Gabriel; Requena Felis, Ángel; Revuelta Arnao, Paula; Such Savall, Cristian; Talens Payá, Adrián; Vega Rubio, Tamara; Verdú Calvo, Carlos; López Martínez, Juan; Rayón Encinas, Emilio.
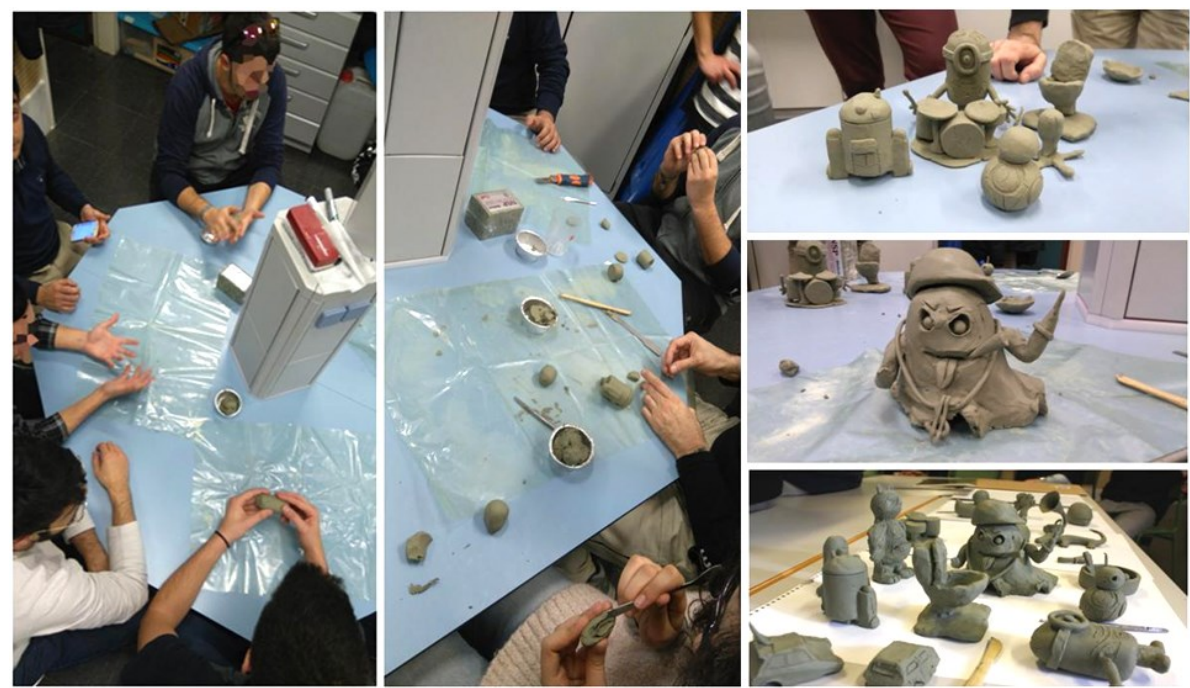

Figura 5.Fotografias que muestran a los estudiantes trabajando en equipo para realizar modelos de prototipos utilizando arcilla de modelar profesional

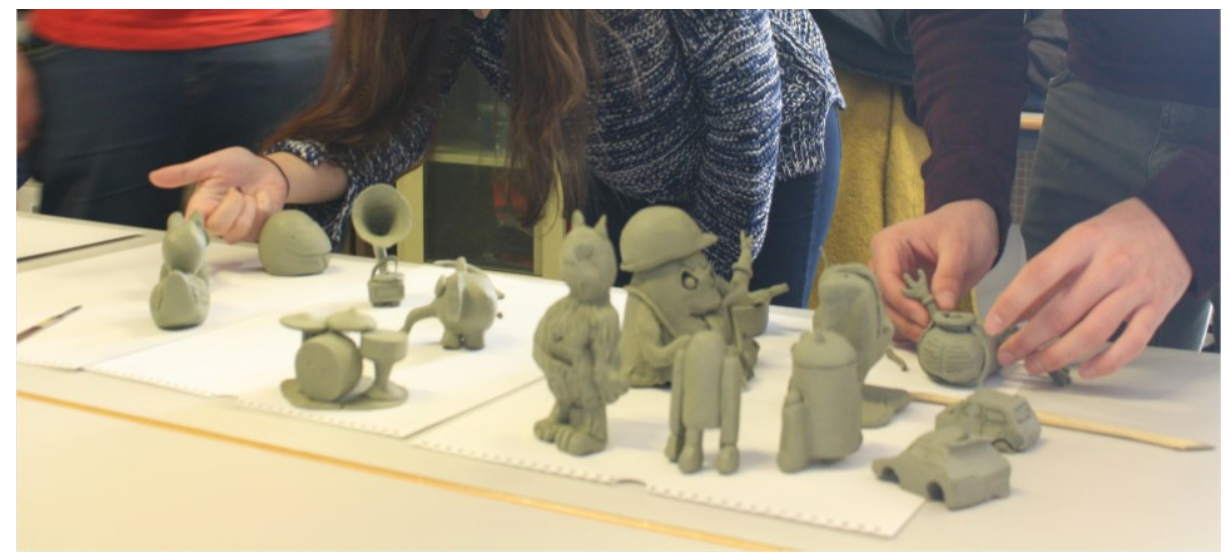

Figura 6. Algunos de los prototipos realizados de forma individual utilizando arcilla de modelado profesional

Otra de las ventajas de modelar en arcilla es la de poder escanear la figura mediante un escáner 3D para tener el objeto en un modelo CAD. Este proceso lo llevamos a cabo en uno de los laboratorios que cuenta con un escáner 3D de luz estructurada, figura 7, que permite obtener una malla de puntos con la que poder trabajar con software específico de modelado 3D digital que además conocemos de otras asignaturas de la carrera.

Figura 10. Figuras realizadas con arcilla termoestable.

(cc) EY-NC-ND 2016, Universitat Politècnica de València

Congreso In-Red (2016) 

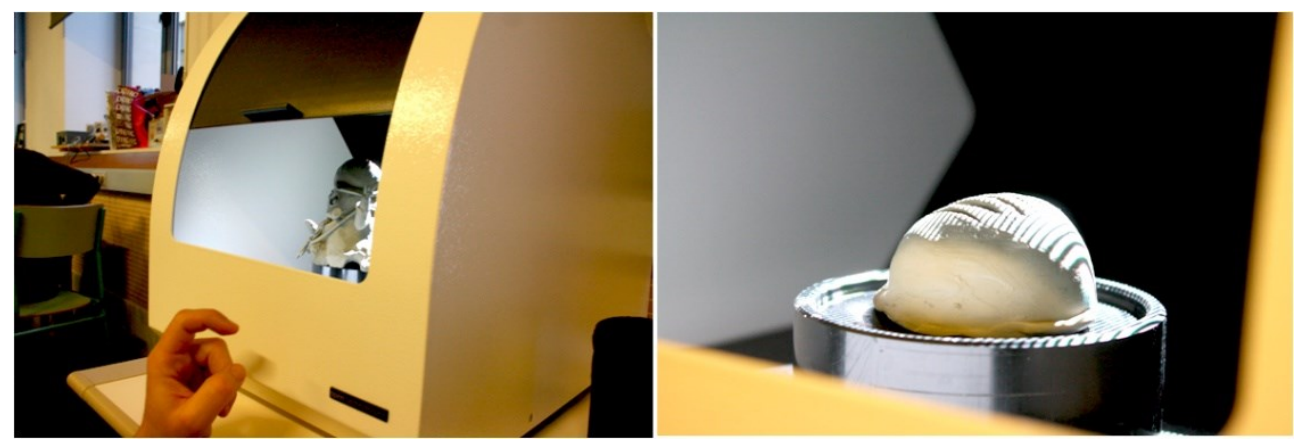

Figura 7. Utilizando un escáner 3D de luz estructurada para digitalizar las superficies de las piezas modeladas.

Con el modelo digitalizado mediante el escáner los estudiantes utilizaron programas informáticos dedicados para cambiar la apariencia de este objeto, realizando simulaciones de diferentes colores, texturas, materiales, etc.

Por otro lado, con el mismo modelo en arcilla es posible también obtener un prototipo al que se le puede añadir mecanismos motorizados, luces, botones, sensores, etc. Según el proyecto propuesto para realizar en equipos, se desarrolló el modelo de un juguete basado en la cabeza de Homer Simpson dotándole de movilidad en los labios y luces en los ojos, que se activaban según las condiciones de juego establecidas en grupo, figuras 8 y 9 .

Para dotar al prototipo de la tecnología necesaria para dotarlo de movimiento, luces y sonido, recurrimos a un sistema desarrollado en el MIT que se explica como por un pequeño ordenador del tamaño de una caja de cerillas y por un precio de menos de 30 euros. Este sistema electrónico es capaz de detectar sensores o inputs, computar con esta información y como resultado activar motores, luces, sonido o cualquier cosa que la imaginación y talento de nuestros estudiantes sean capaces de plantear, figura 10. Otra ventaja de este sistema es que no se requieren conocimientos avanzados de electrónica y al ser un sistema 'open source' existen plataformas en internet donde se comparte toda la información necesaria para crear cualquier tipo de proyecto sin conocimientos previos. Otra placa basada en el mismo sistema pero dedicada a generar sonidos y reproducir cualquier tipo de fichero mp3 o sonido MIDI que pueda descargarse de internet fue suministrada también a los estudiantes. Estas placas están alimentadas mediante una entrada USB y necesita conectarse a un altavoz para reproducir sonidos. El sistema de Arduino causó una gran impresión al descubrir, cada uno dentro de sus necesidades e ideas, un gran abanico de posibilidades para aplicarlo $\mathrm{s}$ sus prototipos de PFG o a proyectos que fuera del ámbito universitario estaban desarrollando. 
Aroca Martinez, Antonio; Calles Díaz, Héctor; Chisbert Victory, Daniel; Cremades Navarro, Miguel Ángel; Diago De Rozas, Rodrigo; Esplugues Calabuig, David; Ferez Navarro, Mario; González

Simón, Francisco Javier; Morera Gilabert, Óscar; Munguía Lozano, María Fernanda; Ortiz Castillo, Álvaro; Ortuño Ayuso, Pablo; Pérez Montoya, Sergio; Popescu, Gabriel; Requena Felis, Ángel; Revuelta Arnao, Paula; Such Savall, Cristian; Talens Payá, Adrián; Vega Rubio, Tamara; Verdú Calvo, Carlos; López Martínez, Juan; Rayón Encinas, Emilio.
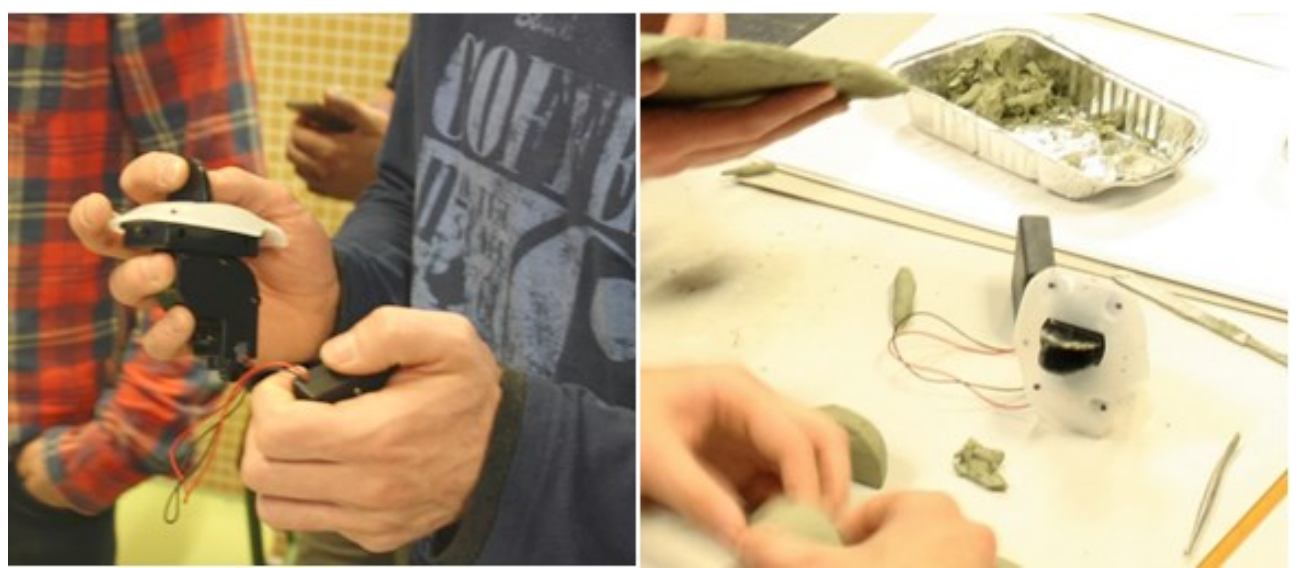

Figura 8. Módulo electrónico motorizado utilizado para dotar de movimiento el modelo de arcilla.
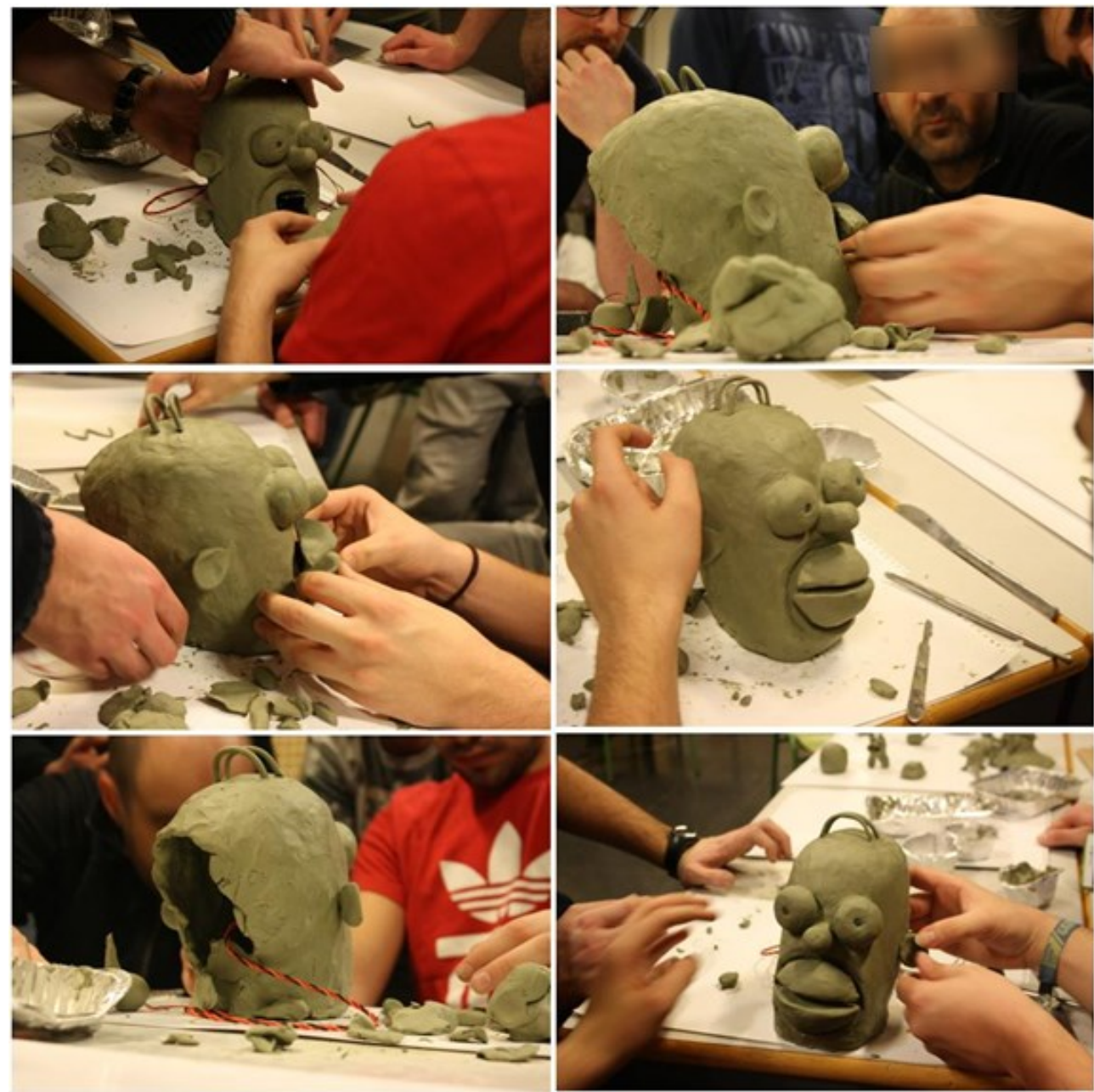

Figura 9. Modelado del prototipo al que se le unen elementos motorizados y luminosos para hacerlo funcional.

(cc) EY-NC-ND 2016, Universitat Politècnica de València

Congreso In-Red (2016) 
Proyecto docente innovador desarrollado en Grado en Ingeniería en Diseño Industrial y Desarrollo de Producto para la consecución de un prototipo funcional mediante trabajo en equipo

Para hacer aún más atractivo si cabe el prototipo se experimentó con una pintura conductora de electricidad, que es un acetato de macropartículas metálicas conductoras, papel de aluminio, cartulina, pinceles, y pinzas para circuitos eléctricos. Todo este trabajo se realizó en equipo como muestra las fotografías de la figura 11. La pintura conductora fue aplicada sobre diferentes superficies e incluso se realizaron dibujos que actuaron como detector de proximidad para que la cabeza de juguete emitiera diferentes sonidos. Al mismo tiempo que algunos compañeros se dedican a pintar sobre una cartulina distintos bocetos, otros se dedican a descargar de internet sonidos en formato $\mathrm{mp} 3$ relacionados con los dibujos plasmados. Una vez seleccionados los sonidos se guardan en una tarjeta micro SD que se inserta en la placa electrónica.

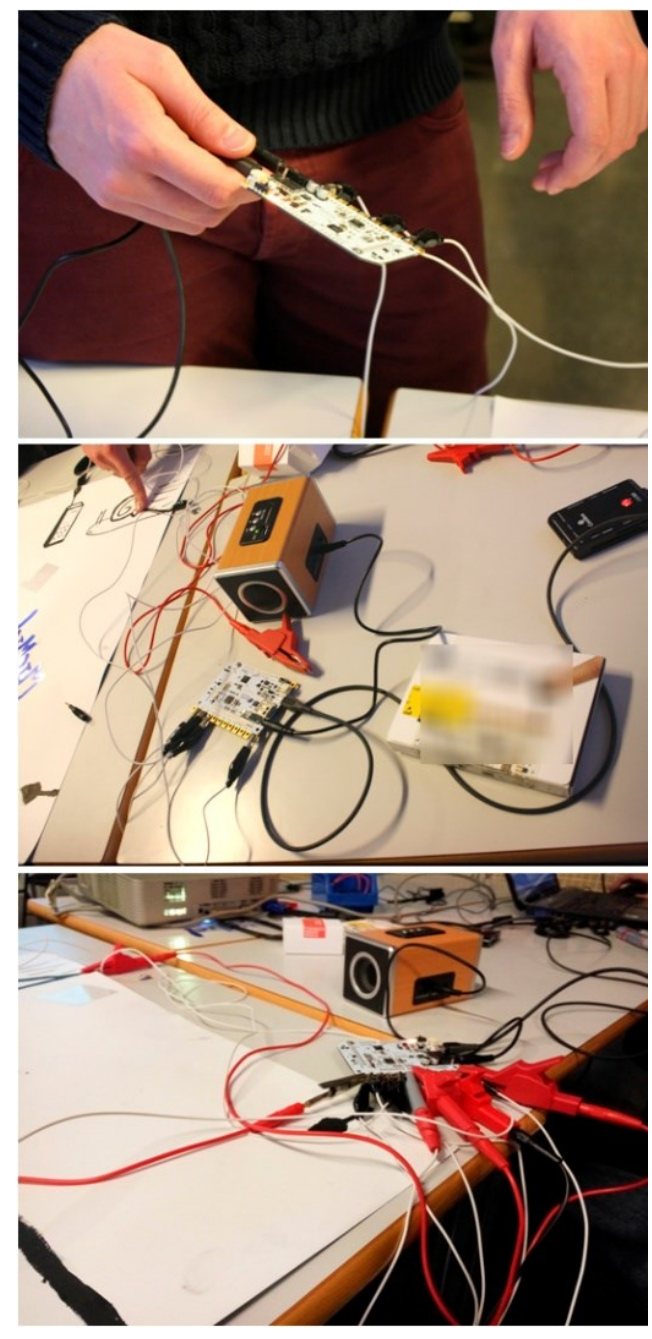

Figura 10. Tecnología desarrollada en el MIT utilizada en clase para funcionalizar el modelo arcilla. 
Aroca Martinez, Antonio; Calles Díaz, Héctor; Chisbert Victory, Daniel; Cremades Navarro, Miguel Ángel; Diago De Rozas, Rodrigo; Esplugues Calabuig, David; Ferez Navarro, Mario; González

Simón, Francisco Javier; Morera Gilabert, Óscar; Munguía Lozano, María Fernanda; Ortiz Castillo, Álvaro; Ortuño Ayuso, Pablo; Pérez Montoya, Sergio; Popescu, Gabriel; Requena Felis, Ángel; Revuelta Arnao, Paula; Such Savall, Cristian; Talens Payá, Adrián; Vega Rubio, Tamara; Verdú Calvo, Carlos; López Martínez, Juan; Rayón Encinas, Emilio.

Una vez que todo está conectado, simplemente hay que tocar los dibujos para que se reproduzca automáticamente el sonido que fue descargado de internet, a la vez que la cabeza movía la boca y encendía las luces de sus ojos.

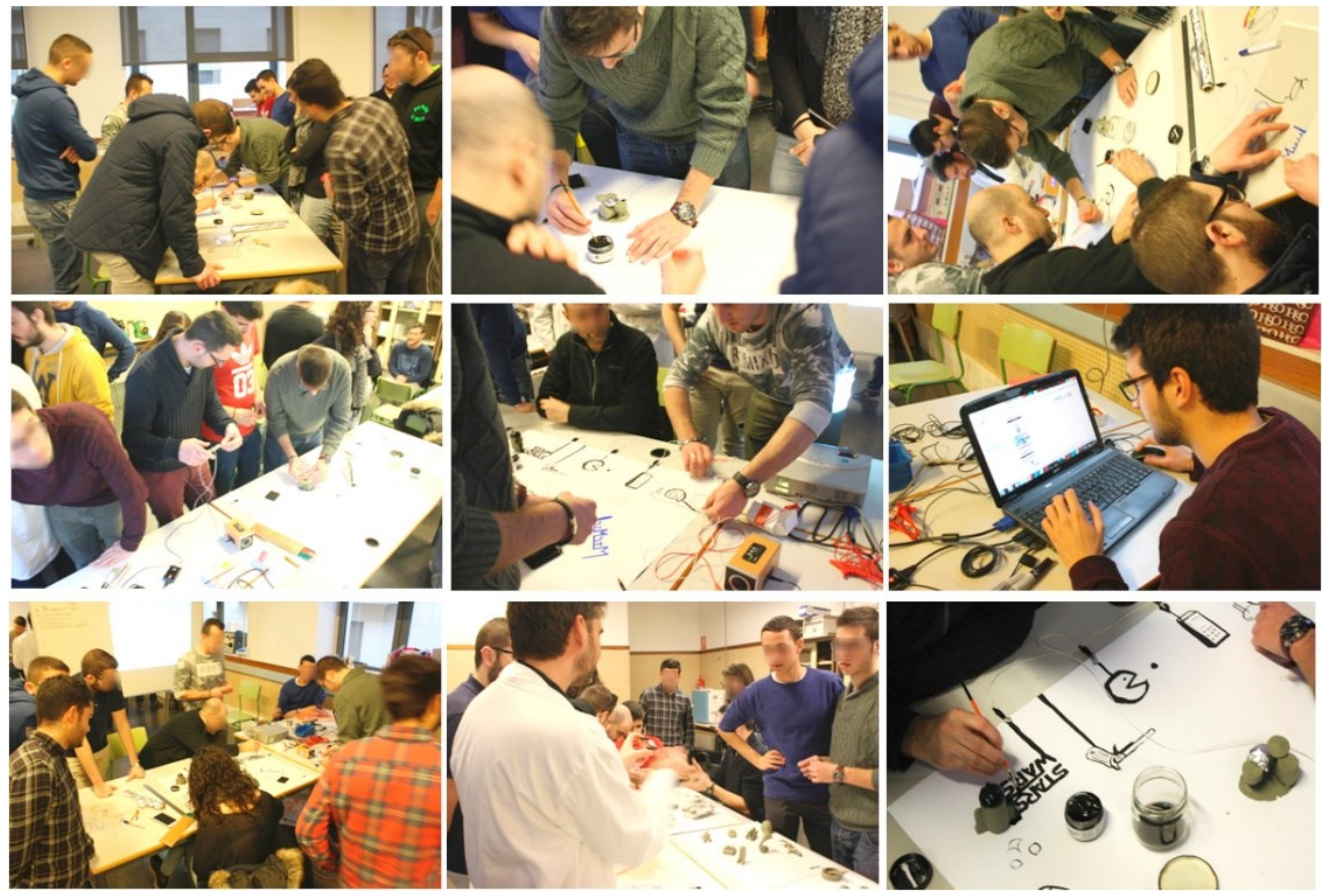

Figura 10. Estudiantes trabajando en equipo con las diferentes tareas requeridas para alcanzar a desarrollar un prototipo funcional.

\section{Conclusiones}

Mediante este procedimiento se ha conseguido dar a conocer a los estudiantes diversos métodos, tecnologías y materiales de actualidad que les pueden servir a lo largo de su carrera profesional. Durante el desarrollo del método docente, se ha fomentado y favorecido la toma de decisiones en equipo dentro de un grupo de trabajo. De manera colateral se ha fomentado un espíritu creativo trabajando con técnicas y herramientas como la de modelar en arcilla para posteriormente utilizar un escáner 3D, o la de utilizar diferentes recursos de luz para luego combinarlos con materiales translúcidos especiales. El hecho de haber alcanzado a desarrollar un modelo de prototipo funcional que interactúe mediante sonidos, movimientos y luces corrobora tanto la eficacia del método como el valor didáctico del mismo.

Nota del profesor: Enhorabuena y gracias a toda la clase de Tecnología del Producto del curso 2015-2016 por los éxitos conseguidos. Ha sido un placer y una satisfacción el haber

(cc) BY-NC-ND 2016, Universitat Politècnica de València

Congreso In-Red (2016) 
Proyecto docente innovador desarrollado en Grado en Ingeniería en Diseño Industrial y Desarrollo de Producto para la consecución de un prototipo funcional mediante trabajo en equipo

compartido con vosotros este procedimiento docente que espero haya sido a la vez que innovador, útil y enriquecedor.

\section{Agradecimientos}

El docente quiere aprovechar estas líneas para agradecer a Fernando Monteropinar, Jose Luis Salinas y Enrique Jorge de ThyssenKrupp Plastic Ibérica en Valencia por el suministro de material de Plexiglás con el que pudimos experimentar en esta metodología docente y por su desinteresado y cálido recibimiento en su empresa.

(c) EY-NC-ND 2016, Universitat Politècnica de València 